\title{
DIPHTHERIA: A STATISTICAL STUDY OF CERTAIN LABORATORY AND CLINICAL OBSERVATIONS.*
}

\author{
HENRY ALBERT, \\ Professor of Pathology and Bacteriology, College of Medicine, University of Iowa; Director of the Iowa \\ State Board of Health Bacteriological Laboratory.
}

VITAL statistics relative to diphtheria have been collected from time to time and have proved of great importance. Statistics relative to laboratory findings and their relation to the clinical aspects of the disease have, however, not been so systematically collected and studied, and there are comparatively few tables of much value. Those which are accessible refer almost entirely to urban conditions, very few to rural communities.

The bacteriological laboratory of the Iowa State Board of Health has now been in existence a little more than two years, and during that time has made 6,155 examinations (February 16,1907 ) for the presence of diphtheria bacilli for the physicians of the state. These examinations were made both for diagnosis and for release from quarantine. The conditions upon which release from quarantine is based in Iowa are as follows:

Quarantine shall be released in those houses in which diphtheria has been diagnosed when synchronous cultures taken from the noses and throats of all infected persons quarantined show two consecutive negative examinations, providing the regulations of the board regarding disinfection and fumigation have first been complied with.

Those who have been quarantined with diphtheria patients may be released from quarantine where both nose and throat cultures on examination by the bacteriologist of the State Board of Health do not show the presence of diphtheria bacilli.

In districts where it is not possible or desirable to use the laboratory findings as a means of regulating quarantine, those suffering from diphtheria shall be quarantined for a period of not less than four weeks from initial symptoms where antitoxin is used, and five weeks where antitoxin is not used.

In order that the statistics to which I am about to refer in detail may be properly understood, it should be stated that diagnosis outfits containing culture media are kept in every city and town in Iowa in places called culture or supply stations, from which they may be obtained at any time by physicians. These diagnosis outfits contain

* Received for publication February 20, 1907. 


\section{Diphtheria: Laboratory and Clinical Observations 2 I I}

a card for the collection of statistical data, on which is printed the following:

FRONT.

DIPHTHERLA.

[To be flled out by physician only.]

[See also other side.]
Patient's name. . . . . . . . . . . . . . . . . .

Address.

Physician's name.

Address.

Health officer's name

Address
[To be filled out at laboratory only.]

Case No......... Report, majl-telegraph

Diagnosis . . . . . . . . . . . . . . . . .

Received ........

Reported

Examined by.....

Notes

Shall report be sent by mail or telegraph ?......... (Telegraphic reports are at physician's expense.)

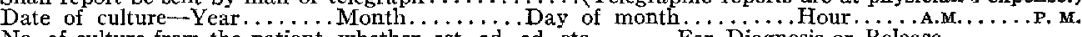

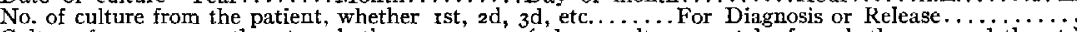
Culture from nose or throat or both........... (release culture must be from both nose and throat.)

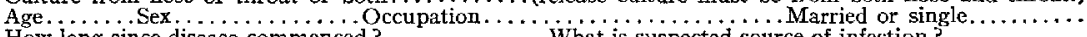

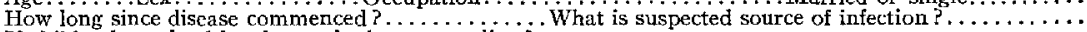

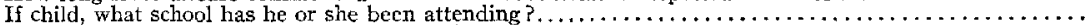

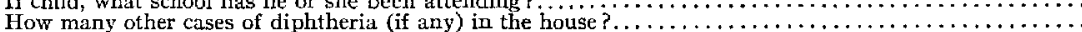
(OVER)

BACK.

[To be filled out by the physician only; see other side also.]

Membrane present . . . . . . Nares. . . . . . . Pharynx. . . . . . Tonsils . . . . . . Larynx. . . . . .

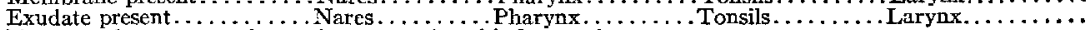

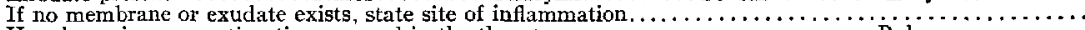
How long since an antiseptic was used in the throat $\ldots \ldots \ldots \ldots \ldots \ldots \ldots \ldots \ldots \ldots \ldots \ldots \ldots \ldots \ldots$

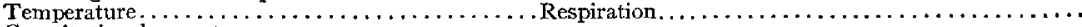

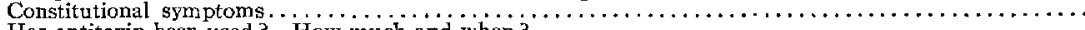

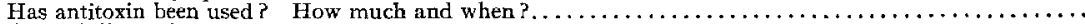

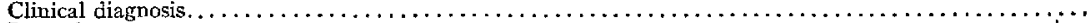

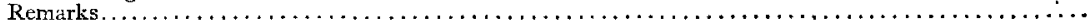

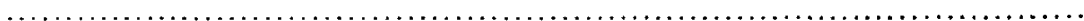

(Please fill out both sides of card as indicated for every preparation made) IOWA STATE BOARD OF HEALTH, BACTERIOLOGICAL LABORATORY, (UNIVERSITY OF IOWA) IOWA CITY, IOWA (OVER)

After the bacteriological examination has been made, the report is sent on a form like that given below:

FRONT.

It is recommended that no culture for release from quarantine be taken prior to fourt een days from date of the development of the disease.

A second culture for release from quarantine should not be taken until report of first culture is received.

IOWA STATE BOARD OF FEALTH, BACTERIOLOGICAL LABORATORY, (UNIVERSITY OF IOWA) IOWA CITY, IOWA

IOWA CitY, IOWA,

Dr.

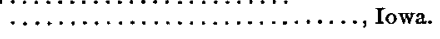

DEAR DOCTOR:

The culture No........... form

submitted by you has been examined.

Diagnosis: .

(see other side)



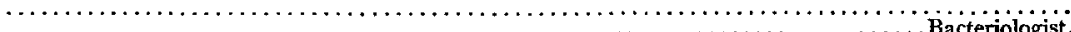

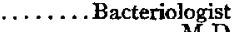

(Every case of diphtheria must be reported to the local Board of Health.)

Director.

(OVER) 
BACK.

\section{INTERPRETATION OF DIAGNOSIS GIVEN.}

1. DIPHTHERIA BACILLI PRESENT, indicates that-

a) The patient has diphtheria or has recently had it; or

b) The diphtheria bacilli are present, even though no lesions of the disease exist (as occasionally occurs, especially among nurses). Such individuals are capable of spreading the disease.

2. NO DIPHTHERIA BACILII FOUND indicates-

a) The entire absence of diphtheria bacilli; or

b) That the culture was not properly made, due to improper technique either in applying swab to the throat or in smearing the swab over the surface of the medium (please carefully follow the instructions); or

c) That some antiseptic was applied to the throat shortly before taking the specimen. It interferes with the subsequent development of the bacteria; or

d) That the infection was in the larynx, and the swab may not have touched the membrane; or

e) That it is one of the rare cases where only a very few diphtheria bacilli are present at the beginning, and may have escaped observation when making the examination. More will be present in a later culture.

If there is any doubt about the case, send us another specimen.

3. QUESTIONABLE may mean-

a) That no growth developed on the media; or

b) That the growth was so scanty that no diagnosis could be made; or

c) That the culture media were not in proper condition-too dry or contaminated. Such media should not be used.

When the specimen is labeled "questionable," it will again be examined the following day. If diphtheria bacilli are found, you will be notified at once; if it is negative, no report will be sent. But do not wait-send another specimen immediately, if you deem advisable.

(OVER)

The following records are based upon reports of examinations made as indicated above. Of these examinations, 2,867 were for diagnosis (Tables $1-6$ ) and 3,063 for release from quarantine (Table 7). Only one culture was submitted for diagnoiss from 2,743 patients, two cultures from 124 patients; I,20I of these were males, I,4I 2 females; 438 of them were married, $I, 639$ unmarried, 7 were widowed. In regard to age, 564 were from one to five years, I,I 66 from six to I 5 years, $76 \mathrm{I}$ from $\mathrm{i} 6$ to 60 years, nine over 60 years.

\section{TABLE I.}

Diagnosis by Physician and Laboratory Findings.

A. Diphtheria (by physician):

Laboratory found diphtheria bacilli . . . . . . . . 670

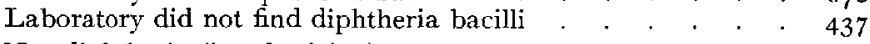

B. Not diphtheria (by physician):

Laboratory found diphtheria bacilli . . . . . . . . 137

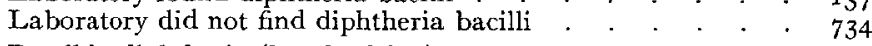

C. Possibly diphtheria (by physician):

Laboratory found diphtheria bacilli . . . . . . . . I5I

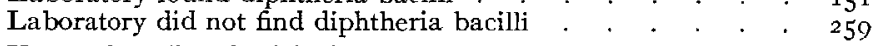

D. Uncertainty (by physician)

Laboratory found diphtheria bacilli . . . . . . . . $\quad 67$

Laboratory did not find diphtheria bacilli . . . . . . . 103 
TABLE 2

Agents

OCCUPation.

Bankers
Barbers
Business (indoors)
Clergymen $\cdot$
Dentists $\cdot$
Druggists $\cdot$
Housekeepers
Laborers (outside)
Lawyers
Mail carriers
Nurses .
Physicians $\cdot$
Railway employees
Pupils and students
Teachers
Veterinary surgeons

, . . , . . . . 4

Barbers

Business (indoors)

Druggists

Laborers (outside)

Lawyers

Mail carriers

Physicians

Railway employees

Teachers

Veterinary surgeons

Lencih of Time after Beginning of Disease that Culture was Sent.

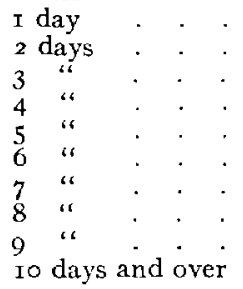

TABLE 4.

Not known

School

Family

Physician

Nurse

TABLE 5 .

Number of Other Cases of Diphtheria in the House.

None
1.
2
3
4
4.

\section{"}

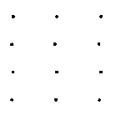

TABLE 6.

A. Membrane (fibrinous exudate) present:

Nares

Pharynx

Larynx

B. Exudate (serous) present:

Nares

Tonsils

C. No membrane, no exudate, but hyperemia 
TABLE 7.

RELEASE From QUARANTINE.

A. Number of specimens sent tó ultain release from quarantine:

I specimen

2 specimens

$$
3
$$

4 ،

6 " 7

B. Release from quarantine in no. of days after beginning of disease:

I5-2 Ist "

$22-28$ th "

$20-3.5$ th "

Table I proves exceedingly interesting and shows that diphtheria bacilli were found in 60 per cent of cases in which the clinical diagnosis of diphtheria was made; in 15 per cent of the cases in which the diagnosis of non-diphtheritic inflammation of the tonsils, pharynx, larynx, or nares was made; in $3^{6}$ per cent of the cases in which the physician made a diagnosis of "possibly diphtheria;" and in 38 per cent of the cases where the diagnosis was questionable in the mind of the physician. It may seem strange that diphtheria bacilli were found in only 60 per cent of the cases in which a clinical diagnosis of diphtheria had been made. This rather low percentage is probably due, to a great extent, to the fact that many of the pseudo-membranous forms of angina caused by streptococci, staphylococci, influenza bacilli, pneumococci, colon bacilli, and the spirochete of Vincent's angina present a picture clinically often indistinguishable from true diphtheria, and for that reason such cases have in many instances been called diphtheria. In many of these cases repeated subsequent examinations have confirmed the first findings. The figures of the Department of Health of the city of Chicago, as given in its biennial report for the year 1904-5, show that diphtheria bacilli were found in only 37 per cent of cases that were clinically diagnosed as diphtheria, in 16 per cent of cases clinically diagnosed as non-diphtheritic, and in 28 per cent of cases in which the clinical diagnosis was not given. In making these calculations it must be remembered that there is a certain percentage of error-an error of technique. Just how great this is we have never been able thoroughly to satisfy ourselves. Our table further emphasizes the fact that the clinical features of diphtheria are such that the laboratory finding is of inestimable value as 
an aid in obtaining a correct diagnosis. The greater discrepancy between the laboratory and clinical findings, as seen in the reports from cities as compared with the figures which we give, may be accounted for by the fact that in cities diphtheria is more or less constantly present. A knowledge of this, together with the constant fear of the possibility of epidemics, causes the city physician to report many cases of simple inflammation of the throat as suspected diphtheria.

Table 2 brings out the fact that $\mathrm{r}, 203$ out of 1,938 cases of diphtheria occurred among school children or among students, and 27 cases among teachers. This may, of course, be explained in large part by the fact that diphtheria occurs more frequently among children. It also, however, suggests the part played by the schoolroom in transmitting disease-a part which is still more emphasized by consideration of Table 4. The rather large number of cases occurring among physicians and nurses, as compared with people in other professions and occupations, is in accordance with what has long been recognized, that diphtheria is one of the most frequent and fatal diseases affecting medical attendants. The fact that in a number of cases physicians and nurses have been found to carry the infection to their patients emphasizes the necessity of the utmost precaution against carrying infection which should be taken by them when visiting and examining diphtheria patients. The use of a cap and gown which may be disinfected after each visit is recommended.

When we consider that in $I, 453$ out of 2,038 cases under observation as shown in Table 4, the source of infection was not known, we must conclude either that diphtheria bacilli may survive some time on various substances, such as dirt and dried sputum, which experiment has proved is rather improbable, or that infection is due to socalled "bacilli carriers"- - people who may or may not have had a previous attack of the disease, but who carry in their throats diphtheria bacilli of sufficient virulence to cause infection in other individuals.' This emphasizes the importance of rules prohibiting expectoration in public, which is no doubt a fertile source of such unknown infections; also the importance of repeated bacteriological examinations to determine the absence of virulent diphtheria bacilli before the release of exposed individuals from quarantine.

r For references on this subject see footnote: Jour. Infect. Dis. 1907, 4, p. 36 . 
In 395 out of 2,038 cases infection was traced directly to the schoolroom. Proper and routine medical inspection of school children, especially when diphtheria is known to exist in a community, is thus exceedingly important. When the disease is at all prevalent among school children, the schools should be closed. I also believe it to be advisable that schoolrooms should be occasionally disinfected when diphtheria exists in a community, because of the danger from the so-called "bacilli-carriers."

It should be said that the cases reported as having no inflammation represent, in a number of instances, members of a family who had been exposed to the disease in which the general symptoms led the physician to suspect the possibility of diphtheria, but where no inflammation existed to account for any local infection.

In the majority of instances, I,505 cases, no antitoxin had been given at the time the specimen was taken for examination, while in $8{ }_{5} 5$ cases it was given. This does not, of course, mean that in all of those instances the physician waited for the report from the laboratory before giving antitoxin. We have found many instances in which no antitoxin was given until the laboratory report was received. This may be considered advisable in some cases when symptoms are very mild. We have always urged, however, that in all cases where diphtheria is suspected and the disease is at all marked, physicians should give antitoxin immediately, the earlier the better, and not wait until the laboratory report is received. During that time the patient may be isolated, and if developments, based upon the laboratory and clinical findings, should prove that diphtheria does not exist, such patient need not of course be quarantined. Instances have occurred in which the patients were charity cases, and physicians, knowing that the cost of the antitoxin must be borne by themselves, have not given the serum until the laboratory reported positive findings. This procedure can scarcely be considered as entirely proper in the interest of the patient, nor yet can the physician be blamed so long as the official authorities continue to cut or refuse to pay bills for charity patients. Provision should be made for the proper compensation of physicians doing charity work; or, better yet, antitoxin should be furnished free to charity patients.

As stated, patients may be released from quarantine when two con- 
secutive examinations of swabs from both the nose and throat indicate the probable absence of diphtheria bacilli. It will be noticed (Table $7 \mathrm{~A}$ ) that many patients have been released on one examination. Many of these represent cases of members of families who have been exposed to diphtheria and who have been released from quarantine upon a single examination taken after a period of incubation of seven days. The period of incubation should be taken into consideration, inasmuch as we cannot necessarily expect to find diphtheria bacilli during that period, and many patients who might later develop the disease would be released from quarantine through a negative result based upon a bacteriological examination within that time. In many instances also the physician has not followed the rule of two consecutive examinations to the letter and has allowed the release upon a single examination. It will readily be understood that in rural communities a strict observation of the law cannot always be maintained. Our table indicates that, in a number of cases, three, four, five, six, and even seven specimens were necessary to obtain release from quarantine. These numbers may be accounted for by the fact that either physicians begin to take the specimens for release very early, and take them in rapid succession, or that the bacteria remain in the throats of these patients an unusually long time.

The old-time rule enforced in this state was that all cases of diphtheria must remain in quarantine for five weeks. This has now been modified to read as follows:

In districts where it is not possible or desired to use the laboratory findings as a means of regulating quarantine, those suffering from diphtheria shall be quarantined for a period of not less than four weeks from initial symptoms where antitoxin is used, and five weeks where antitoxin is not used.

Table 7 also shows that 700 out of 1,682 , or $4 \mathrm{I}$ per cent, were released from quarantine before the end of the second week after the beginning of the disease; 654 , or $3^{2}$ per cent, before three weeks after the beginning of the disease; 279 , or 16 per cent, from three to four weeks after the beginning of the disease; 45 , or 0.02 per cent, from four to five weeks after the beginning of the disease; and that 4 , or 0.002 per cent, were not released from quarantine until after five weeks after the beginning of the disease. The large number of cases of release from quarantine within two weeks after the beginning of 
the disease may be accounted for in part by the number of specimens taken from individuals exposed to the disease, but rclcased from quarantine as soon as a negative report was received from the laboratory; in part by the fact that in a number of cases diphtheria bacilli had entirely disappeared from the nose and throat before the end of the second week after the beginning of the disease; in part, however, it must also be explained by the fact that in a number of cases physicians have taken advantage of the laboratory method of relcasing patients from quarantine, and have so collected the specimens for examination that a negative report was bound to be received, whether diphtheria bacilli were present or not. The rule enforced in some of our larger cities (Des Moines is the only city in this state following such rule) is that the health officer or medical inspector must take all of the specimens for release from quarantine, or at least the last specimen after the physician has received one negative report from the laboratory. This rule is not easily enforced in many of the smaller towns and rural communities. Because of the fact that certain physicians will take advantage of this privilege, the board has passed the following resolutions:

It is recommended that no culture for release from quarantine be taken prior to fourtecn days from the date of the development of the disease.

A second culture for release from quarantine should not be taken until report of first culture is received.

The table is, however, of significance in that it shows that a majority of the cases of diphtheria have been released from quarantine during the second and third week after the beginning of the disease process. When examinations prove the presence of the diphtheria bacilli after five weeks from the date of development of the disease, the virulence test of the microorganism is always made to determine whether the bacteria present are or are not virulent. If found not virulent, the patient may be released from quarantine. 